\title{
Clinical Course and Features of Critical Patients with COVID-19: A Single- Center, Retrospective Study from Wuhan Huoshenshan Hospital
}

Huisi He

Eastern Hepatobiliary Surgery Hospital

Zhichao Jin

Second Military Medical University

Yibin Ren

Second Military Medical University

Junxue Wang

Second Military Medical University

\section{Shuzhen Chen}

Second Military Medical University

Wen Wen

Second Military Medical University

Yushan Miao

Second Military Medical University

Xuewei Qi

CAS: Chinese Academy of Sciences

\section{Taiyu Shang}

Fudan University

Chenxu Zhang

Second Military Medical University

Hongyang Wang

Second Military Medical University

\section{Weiqin Li}

Nanjing University

Xijing Zhang

Fourth Military Medical University

Hao Tang ( $\nabla$ tanghao_0921@126.com )

Changzheng Hospital: Shanghai Changzheng Hospital

Research 
Keywords: SARS-CoV-2, coronavirus, transmission, ARDS, pneumonia, Huoshenshan

Posted Date: October 14th, 2020

DOI: https://doi.org/10.21203/rs.3.rs-89722/v1

License: (1) (1) This work is licensed under a Creative Commons Attribution 4.0 International License. Read Full License 


\section{Abstract}

Background: Nationally, the indicators tracking the coronavirus pandemic has remained stable. However, it's still a public health concern and it's worth providing more front-line data on critical illness. We aim to investigate the clinical course and features of critical patients with Corona Virus Disease 2019 (COVID19).

Methods: The data on 124 consecutive critical patients from $8^{\text {th }}$ February through April $16^{\text {th }} 2020$, including demographic and clinical information, were obtained from the intensive care unit (ICU) of Wuhan Huoshenshan Hospital. A cross-sectional survey and comparisons of key biomarkers between survivors and nonsurvivors were performed.

Results: Over the study period, 57 nonsurvivors and 67 survivors were included. The overall case-fatality rate for critical patients with COVID-19 was approximately $46 \%$. The overall average age was $69.89 \pm 11.03$ years, and the majority had underlying health problems such as hypertension (63[51\%]) and diabetes (27[22\%]). Compared with survivors, nonsurvivors were more likely to develop sepsis (57[100\%] vs. $34[51 \%])$, acute respiratory distress syndrome (52[91\%] vs. $21[38 \%])$ and organ dysfunction. Besides, the dynamic changes in some biomarkers (i.e. WBC, TLC, CRP, PLT) were significantly different between the two groups. The trajectories of temperature revealed that the group with a high temperature on admission that steadily declined had the highest percentage of deaths (84.21\%).

Conclusions: The elderly with many concomitant diseases were at the highest risk. Lymphocyte, platelet, C-reactive protein and temperature were revealed to have potential as prognostic factors, whereas some other biomarkers, such as hepatic enzymes, may not offer additional information. Moreover, patients with high temperatures on admission should receive extra care.

\section{Introduction}

A new outbreak of Corona Virus Disease 2019 (COVID-19) occurred in late December 2019 in Wuhan, China, and has spread to other countries with cases doubling within days through person-to-person transmission, which has been confirmed both in and outside of China. ${ }^{1,2}$ As of 1 st October or roughly ten months into the outbreak, the number of cases of COVID-19 worldwide has exceeded 34 million across 215 countries, and China has confirmed 91,069 cases. ${ }^{3}$ Although the viral pandemic has been controlled in China now and indicators tracking its activity continue to be stable, largely due to aggressive public health measures, COVID-19 is still a critical global issue.

According to the World Health Organization (WHO) interim guidelines ${ }^{4}$ and Chinese Clinical Guidance For COVID-19 Pneumonia Diagnosis and Treatment (7th edition) ${ }^{5}$, the confirmed patients are divided into four categories ranging from mild to critical, based on their clinical manifestations. Most studies have investigated COVID-19 at the very beginning, providing well-rounded, detailed illustrations of the clinical characteristics of infected patients. ${ }^{1,2,6-10}$ However, there are certain drawbacks associated with the 
sample sizes and study of critically ill patients who require the greatest use of medical sources. These findings indicate an urgent need to understand the clinical features of COVID-19 that exist among patients in critical condition.

In this study, we sought to analyze data from the intensive care unit (ICU) in Wuhan Huoshenshan Hospital, an emergency specialty field hospital constructed in response to the COVID-19 outbreak and runs under the management of the People's Liberation Army, to help address these research gaps and provide one of the first comprehensive clinical descriptions of critical patients with COVID-19 from the first anti-pandemic frontline.

\section{Materials And Methods}

\subsection{Data source}

This was a cross-sectional study focused on critical patients with COVID-19. We included all critically ill patients with COVID-19 registered in the ICU of Wuhan Huoshenshan Hospital from February 8th, the day this emergency specialty field hospital was put into use, to April 16th, when the hospital announced its closure.

\subsection{Participants \& Ethical consideration}

Patients were included if they met the following criteria: 1) primary diagnosis of COVID-19 confirmed by next-generation sequencing or real-time polymerase-chain-reaction methods; 2 ) defined as critical based on the need for supportive care with mechanical ventilation or treatment for organ failure. And among those patients who were admitted to ICU twice, the second one was taken.

Informed consent was not obtained from the patients for this study due to the ongoing viral epidemic in mainland China during that period. This study was approved by the Ethics Commission of Wuhan Huoshenshan Hospital. (No. IEC-AF-SC-05)

\subsection{Data collection}

Demographic and clinical data were retrieved from electronic medical records and nursing records, including gender, sex, onset symptoms, underlying health problems, vital signs on admission, baseline laboratory data before treatment (i.e., blood routine, liver function, kidney function, electrolyte levels, coagulation function, cardiac function, $\mathrm{C}$ reactive protein [CRP], procalcitonin [PCT]), dynamic changes in key laboratory indexes (i.e., temperature, white blood cell count [WBC], total lymphocyte count [TLC], platelet [PLT], C-reactive protein [CRP], alanine transaminase [ALT], aspartate transaminase [AST], PCT, total bilirubin [TBIL], creatinine [CRE], creatine kinase-MB [CK-MB]), comorbidities and complications, and treatments. Data collection was completed by three independent authors.

\subsection{Outcomes}


The primary endpoint of interest was fatality after ICU admission. Those diagnosed as recovered patients were evaluated according to the Novel Coronavirus Pneumonia Diagnosis and Treatment Plan (provisional, version 7.0). ${ }^{5}$ Additionally, the follow-up was defined as the period from admission into the ICU until meeting the primary endpoint.

The secondary endpoints were diverse laboratory indexes and the incidence of complications. Acute respiratory disease syndrome (ARDS) was recognized based on the Berlin ARDS definition. ${ }^{11}$ Acute kidney injury was diagnosed if CRE increased by $26.5 \mathrm{mmol} / \mathrm{L}$ or more in $48 \mathrm{~h}$ or rose by at least 1.5 -fold from baseline within 7 days. ${ }^{11}$ The diagnosis of gastrointestinal hemorrhage was based on complaints from the patients or aspiration of gastric contents. The term myocardial injury was used when troponin values were elevated above the $99 \%$ upper reference limit. Secondary bacterial infection was detected by symptoms, signs and blood tests (i.e., blood routine, CRP and PCT). The Sequential Organ Failure Assessment (SOFA) score was used to assess the patient's condition to diagnose sepsis (SOFA $\geq 2){ }^{12}$ Coagulopathy was defined as a 3-second extension of the prothrombin time or a 5-second extension of the activated partial thromboplastin time. COVID-19-associated abnormal liver function was diagnosed if ALT or AST increased to 3-fold over the upper limit or if TBIL was more than twice the upper limit without another cause. ${ }^{13}$

\subsection{Statistical analysis}

Descriptive statistics were used to tabulate these patients' clinical features; means and standard deviation or median and interquartile ranges (IQR) are reported for continuous variables, and number is reported for categorical variables. Differences between the survivors and nonsurvivors groups were compared by the $\chi 2$ test, Wilcoxon rank-sum tests and Fisher's exact tests were applied for categorical variables, and t-tests, Wilcoxon rank-sum tests were applied for continuous variables. The distributions were considered significantly different with an $\alpha$ risk of $5 \%$ if the P-value was less than 0.05 .

In addition, we adopted a group-based trajectory modeling approach to identify different patterns of temperature from admission to day 28 (temperature was recorded 6 times each day at 2 a.m., 6 a.m., 10 a.m., 2 p.m., 6 p.m. and 10 p.m.). The shapes and the optimal number of groups were determined by the following criteria: 1) Bayesian information criterion was highest; 2 ) the number of patients in each group was not less than $10 \%$ of the total; 3 ) high average posterior probability of assignment $(>0.7)$.

Data management and analysis were performed using SAS software (version 9.4) for Windows.

\section{Results}

\subsection{Epidemiological data}

Between 8th February and 16th April, 126 patients were admitted to the ICU of Wuhan Huoshenshan Hospital according to the records. Among them, two patients had two records, of which the second one was retained as the basis for endpoint. Ultimately, 124 critical patients met our inclusion criteria and were 
included in this study. (Fig. 1) All 124 patients reached the endpoint with full medical records. Overall, 57 (46\%) of them eventually died, another 67 (54\%) patients survived.

An overview of the baseline characteristics of nonsurvivors $(n=57)$ and survivors $(n=67)$ in terms of demographic and underlying health problems is provided in Table 1. There were 81 (65\%) males and 43 $(35 \%)$ females, and the average age was $69.89 \pm 11.03$ years. We found that the average age was significantly higher in nonsurvivors than in survivors (71.12 \pm 9.24 vs. $68.85 \pm 12.33$ ), which can also be observed visually in Table 1. Closer inspection of this table showed that more than half of the patients had underlying health problems, of which the first few were hypertension (63 [51\%]), diabetes mellitus (27 [22\%]) and cardiovascular diseases (23 [19\%]). Moreover, the most frequently observed symptom was fever (83 [67\%]), followed by dry cough (69 [56\%]) and fatigue (60 [48\%]). Interestingly, few patients developed infrequent conditions, such as headache (4 [3\%]), diarrhea (4 [3\%]) and vomiting (2 [2\%]). 
Table 1

Baseline characteristics for critical patients with COVID-19

\begin{tabular}{|c|c|c|c|}
\hline & $\begin{array}{l}\text { Overall } \\
(n=124)\end{array}$ & $\begin{array}{l}\text { Non-survivors } \\
(n=57)\end{array}$ & $\begin{array}{l}\text { Survivor } \\
(n=67)\end{array}$ \\
\hline \multicolumn{4}{|l|}{ Gender, n (\%) } \\
\hline Male & $81(65)$ & $40(70)$ & $41(61)$ \\
\hline Female & $43(35)$ & $17(30)$ & $26(39)$ \\
\hline \multicolumn{4}{|l|}{ Age, years } \\
\hline Mean \pm SD & $69.89 \pm 11.03$ & $71.12 \pm 9.24$ & $68.85 \pm 12.33$ \\
\hline \multicolumn{4}{|c|}{ Underlying health problems, $n$ (\%) } \\
\hline Hypertension & $63(51)$ & $25(44)$ & $38(57)$ \\
\hline Diabetes mellitus & $27(22)$ & $14(25)$ & $13(20)$ \\
\hline Cardiovascular disease & $23(19)$ & $14(25)$ & $9(13)$ \\
\hline Urinary system diseases & $16(13)$ & $7(12)$ & $9(13)$ \\
\hline Chronic lung disease & $13(10)$ & $6(11)$ & $7(11)$ \\
\hline Digestive system diseases & $13(10)$ & $5(9)$ & $8(12)$ \\
\hline Malignancy & $8(6)$ & $2(4)$ & $6(9)$ \\
\hline \multicolumn{4}{|l|}{ Symptoms, n (\%) } \\
\hline Fever & $83(67)$ & $39(68)$ & $44(66)$ \\
\hline Dry cough & $69(56)$ & $32(56)$ & $37(55)$ \\
\hline Fatigue & $60(48)$ & $31(54)$ & $29(43)$ \\
\hline Breathlessness & $45(36)$ & $22(39)$ & $23(34)$ \\
\hline Myalgia & $26(21)$ & $13(23)$ & $13(19)$ \\
\hline Chest tightness & $19(15)$ & $6(11)$ & $13(19)$ \\
\hline Headache & $4(3)$ & $2(4)$ & $2(4)$ \\
\hline Diarrhea & $4(3)$ & $2(4)$ & $2(3)$ \\
\hline Vomiting & $2(2)$ & $1(2)$ & $1(1)$ \\
\hline
\end{tabular}

\subsection{Clinical features before treatment}


The clinical features before treatment were summarized in Table 2. Interestingly, many features were significantly different between the two groups, including $\mathrm{SaO}_{2}$, Neutrophil ratio, Lymphocyte ratio, PLT, AST, TBIL, CRE, CK-MB, LDH, PCT, etc. Higher WBC (11.49 \pm 8.51 vs. $8.52 \pm 3.45)$ and lower TLC (0.74 \pm 0.43 vs. $0.90 \pm 0.51$ ) were presented in nonsurvivors while there was no statistical significance. Although the PLT in each group were in the normal range, a lower PLT was observed in nonsurvivors (185.47 \pm 127.79 vs. $224.34 \pm 99.64)$. The CRP level, cardiac function and PCT level and coagulation function in nonsurvivors were more severely impaired than those in survivors as well, while most liver function and kidney function remained normal or mildly abnormal. 
Table 2

Basic data of vital signs on admission and laboratory tests before treatments

$\begin{array}{lll}\text { Non-survivors } & \text { Survivor } & \text { P-value } \\ (n=57) & (n=67) & \end{array}$

\section{Vital signs}

\begin{tabular}{llll}
$\mathrm{T},{ }^{\circ} \mathrm{C}$ & $36.94 \pm 0.74$ & $36.66 \pm 0.53$ & $0.0137^{*}$ \\
\hline $\mathrm{SBP}, \mathrm{mmHg}$ & $131.35 \pm 19.98$ & $133.24 \pm 19.37$ & 0.5950 \\
\hline $\mathrm{DBP}, \mathrm{mmHg}$ & $76.88 \pm 15.08$ & $79.78 \pm 13.41$ & 0.2595 \\
\hline $\mathrm{RR}, \mathrm{n}$ & $22(20,24)$ & $21(19,24)$ & 0.3585 \\
\hline $\mathrm{P}, \mathrm{n}$ & $89(81,98)$ & $87(78,98)$ & 0.5222 \\
\hline $\mathrm{SaO} 2, \%$ & $92.28(7.09)$ & $96.42(3.31)$ & $<0.0001^{*}$
\end{tabular}

\section{Laboratory Tests}

\section{Complete Blood Cell}

\begin{tabular}{llll} 
WBC, ${ }^{*} 10^{\wedge} 9 / \mathrm{L}$ & $11.49 \pm 8.51$ & $8.52 \pm 3.45$ & 0.1189 \\
\hline Neutrophil ratio, \% & $85.65 \pm 10.19$ & $80.62 \pm 10.30$ & $0.0025^{*}$ \\
\hline Lymphocyte ratio, \% & $9.05 \pm 7.71$ & $12.24 \pm 8.01$ & $0.0063^{*}$ \\
\hline TLC, ${ }^{*} 10^{\wedge} 9 / \mathrm{L}$ & $0.74 \pm 0.43$ & $0.90 \pm 0.51$ & 0.0749 \\
\hline $\mathrm{RBC},{ }^{*} 10^{\wedge} 12 / \mathrm{L}$ & $3.84 \pm 0.65$ & $3.82 \pm 0.73$ & 0.7906 \\
\hline $\mathrm{HGB}, \mathrm{g} / \mathrm{L}$ & $119.11 \pm 19.70$ & $116.14 \pm 22.86$ & 0.4816 \\
\hline $\mathrm{PLT},{ }^{*} 10^{\wedge} 12 / \mathrm{L}$ & $185.47 \pm 127.79$ & $224.34 \pm 99.64$ & $0.0120^{*}$
\end{tabular}

\section{Liver Function}
$A L T, I U / L$
$40.61 \pm 37.55$
$35.49 \pm 32.01$
0.2805

Data presented as Mean \pm SD or Median (Q1, Q3)

Abbreviation: T, Temperature; SBP, Systolic blood pressure; DBP, Diastolic blood pressure; RR, Respiratory rate; P, Pulse; SaO2, Oxygen Saturation; WBC, White blood cell count; TLC, Total lymphocyte count; RBC, Red blood cell count; HBG, Hemoglobin; PLT, Platelet; CRP, C-reactive protein; ALT, Alanine transaminase; AST, Aspartate transaminase; TBIL, Total bilirubin; TA, Total bile acid; ALP, Alkaline phosphatase; GGT, Gamma-glutamyl transferase; CRE, Creatinine; CK-MB, Creatine kinaseMB; LDH, Lactate dehydrogenase; BNP, B-type natriuretic peptide; hsTNI, hypersensitive troponin I; PT, Prothrombin; INR, International Normalized Ratio; APTT, Activated partial thromboplastin time; PCT, Procalcitonin

*P $<0.05$ for comparison with survivor and non-survivor 


\begin{tabular}{|c|c|c|c|}
\hline & $\begin{array}{l}\text { Non-survivors } \\
(n=57)\end{array}$ & $\begin{array}{l}\text { Survivor } \\
(n=67)\end{array}$ & P-value \\
\hline AST, IU/L & $48.11 \pm 45.20$ & $32.61 \pm 28.63$ & $0.0456^{*}$ \\
\hline Albumin, g/L & $31.69 \pm 4.56$ & $32.62 \pm 4.18$ & 0.4488 \\
\hline TBIL, $\mu \mathrm{mol} / \mathrm{L}$ & $19.08 \pm 20.16$ & $10.35 \pm 4.95$ & $0.0003^{*}$ \\
\hline TBA, $\mu \mathrm{mol} / \mathrm{L}$ & $6.74 \pm 8.19$ & $3.57 \pm 4.01$ & $0.0205^{\star}$ \\
\hline ALP, IU/L & $97.74 \pm 40.46$ & $89.50 \pm 48.57$ & 0.1092 \\
\hline GGT, IU/L & $67.11 \pm 51.73$ & $62.65 \pm 59.07$ & 0.8906 \\
\hline \multicolumn{4}{|l|}{ Kidney Function } \\
\hline UREA, mmol/L & $9.32 \pm 6.06$ & $8.61 \pm 6.73$ & 0.3995 \\
\hline CRE, $\mathrm{mmol} / \mathrm{L}$ & $96.84 \pm 71.44$ & $123.91 \pm 223.03$ & $0.0461^{*}$ \\
\hline \multicolumn{4}{|l|}{ Electrolytes } \\
\hline $\mathrm{K}^{+}, \mathrm{mmol} / \mathrm{L}$ & $4.28 \pm 0.58$ & $4.22 \pm 0.72$ & 0.3491 \\
\hline $\mathrm{Na}^{+}, \mathrm{mmol} / \mathrm{L}$ & $141.68 \pm 8.62$ & $139.96 \pm 4.96$ & 0.8202 \\
\hline \multicolumn{4}{|c|}{ Cardiac function } \\
\hline CK-MB, IU/L & $20.77 \pm 19.61$ & $14.42 \pm 10.99$ & $0.0211^{\star}$ \\
\hline $\mathrm{LDH}, \mathrm{IU} / \mathrm{L}$ & $438.04 \pm 162.74$ & $296.48 \pm 122.92$ & $<0.0001^{*}$ \\
\hline BNP, $\mathrm{pg} / \mathrm{ml}$ & $309.40 \pm 403.34$ & $182.48 \pm 326.95$ & 0.0601 \\
\hline \multicolumn{4}{|c|}{ Coagulation function } \\
\hline PT, s & $16.38 \pm 3.41$ & $14.10 \pm 2.22$ & $0.0006^{*}$ \\
\hline INR, n & $1.37 \pm 0.28$ & $1.17 \pm 0.19$ & $0.0005^{\star}$ \\
\hline
\end{tabular}

Data presented as Mean \pm SD or Median (Q1, Q3)

Abbreviation: T, Temperature; SBP, Systolic blood pressure; DBP, Diastolic blood pressure; RR, Respiratory rate; P, Pulse; SaO2, Oxygen Saturation; WBC, White blood cell count; TLC, Total lymphocyte count; RBC, Red blood cell count; HBG, Hemoglobin; PLT, Platelet; CRP, C-reactive protein; ALT, Alanine transaminase; AST, Aspartate transaminase; TBIL, Total bilirubin; TA, Total bile acid; ALP, Alkaline phosphatase; GGT, Gamma-glutamyl transferase; CRE, Creatinine; CK-MB, Creatine kinaseMB; LDH, Lactate dehydrogenase; BNP, B-type natriuretic peptide; hsTNI, hypersensitive troponin l; PT, Prothrombin; INR, International Normalized Ratio; APTT, Activated partial thromboplastin time; PCT, Procalcitonin

*P $<0.05$ for comparison with survivor and non-survivor 


\begin{tabular}{|c|c|c|c|}
\hline & $\begin{array}{l}\text { Non-survivors } \\
(n=57)\end{array}$ & $\begin{array}{l}\text { Survivor } \\
(n=67)\end{array}$ & P-value \\
\hline APTT, s & $31.60 \pm 9.06$ & $29.70 \pm 5.28$ & 0.6988 \\
\hline D-dimer mg/L & $4.24 \pm 4.41$ & $3.61 \pm 4.71$ & 0.3788 \\
\hline CRP, mg/L & $40.98 \pm 48.75$ & $30.69 \pm 41.99$ & 0.3217 \\
\hline PCT, ng/ml & $0.96 \pm 2.31$ & $0.30 \pm 0.78$ & $0.0018^{*}$ \\
\hline \multicolumn{4}{|c|}{ Data presented as Mean \pm SD or Median (Q1, Q3) } \\
\hline \multicolumn{4}{|c|}{$\begin{array}{l}\text { Abbreviation: T, Temperature; SBP, Systolic blood pressure; DBP, Diastolic blood pressure; RR, } \\
\text { Respiratory rate; P, Pulse; SaO2, Oxygen Saturation; WBC, White blood cell count; TLC, Total } \\
\text { lymphocyte count; RBC, Red blood cell count; HBG, Hemoglobin; PLT, Platelet; CRP, C-reactive protein; } \\
\text { ALT, Alanine transaminase; AST, Aspartate transaminase; TBIL, Total bilirubin; TA, Total bile acid; ALP, } \\
\text { Alkaline phosphatase; GGT, Gamma-glutamyl transferase; CRE, Creatinine; CK-MB, Creatine kinase- } \\
\text { MB; LDH, Lactate dehydrogenase; BNP, B-type natriuretic peptide; hsTNI, hypersensitive troponin I; PT, } \\
\text { Prothrombin; INR, International Normalized Ratio; APTT, Activated partial thromboplastin time; PCT, } \\
\text { Procalcitonin }\end{array}$} \\
\hline \multicolumn{4}{|c|}{ * $P<0.05$ for comparison with survivor and non-survivor } \\
\hline
\end{tabular}


Table 3

Clinical manifestation, including symptoms, complications and treatments of these patients

$\begin{array}{llll}\text { Overall } & \text { Non-survivors } & \text { Survivor } & \text { P-value } \\ (n=124) & (n=57) & (n=67)\end{array}$

\section{The first apartment, $\mathrm{n}(\%)$}

ICU

Non-ICU

\section{Treatments}

Non-invasive ventilation

Invasive ventilation

ECMO

Glucocorticoids

Antiviral drugs

Arbidol hydrochloride

Lianhua Qingwen

Interferon

Ribavirin

Hydroxychloroquine

CBPT

Convalescent plasma

Complication, $\mathrm{n}(\%)$

Sepsis

ARDS

Secondary bacterial infection

Myocardial injury

AKI
$81(65) \quad 40(70)$

$43(35)$

$17(30)$

$65(52)$

$47(82)$

$50(88)$

$5(9)$

$46(81)$

$84(68)$

$76(61)$

$71(57)$

$16(13)$

$13(10)$

8 (10)

$18(15)$

$17(14)$

$96(73)$

$57(100)$

$52(91)$

$42(74)$

$29(51)$

$37(65)$

4 (7)

$5(9)$

$73(59)$

$59(48)$

$40(32)$

$46(37)$
$41(61)$

$26(39)$
$0.0251^{*}$

9 (13)

0.2452

7 (10)

0.1102

$3(4)$

$0.0006^{\star}$

$12(18)$

0.1403

$34(51)<0.0001^{*}$

$21(38)<0.0001^{*}$

$17(25)<0.0001^{*}$

$11(17)<0.0001^{*}$

$9(13)$

Abbreviation: ARDS, acute respiratory distress syndrome AKI, acute kidney injury ECMO, Extracorporeal membrane oxygenation; CBPT, Continuous blood purification therapy

${ }^{*} \mathrm{P}<0.05$ for comparison with survivor and non-survivor 


\begin{tabular}{|lllll}
\hline & $\begin{array}{l}\text { Overall } \\
(\mathbf{n = 1 2 4 )}\end{array}$ & $\begin{array}{l}\text { Non-survivors } \\
(\mathbf{n}=\mathbf{5 7})\end{array}$ & $\begin{array}{l}\text { Survivor } \\
(\mathbf{n = 6 7 )}\end{array}$ & P-value \\
\hline Coagulopathy & $39(31)$ & $35(55)$ & $4(6)$ & $<0.0001^{*}$ \\
\hline Abnormal liver function & $37(30)$ & $29(51)$ & $8(12)$ & $<0.0001^{*}$ \\
\hline Gastrointestinal hemorrhage & $14(11)$ & $9(7)$ & $5(8)$ & 0.1442 \\
\hline
\end{tabular}

Abbreviation: ARDS, acute respiratory distress syndrome AKI, acute kidney injury ECMO, Extracorporeal membrane oxygenation; $\mathrm{CBPT}$, Continuous blood purification therapy

$\star \mathrm{P}<0.05$ for comparison with survivor and non-survivor

\subsection{Findings during clinical course}

As a whole, patients who were transferred to ICU had a higher fatality rate than those received treatments in ICU at the very start (49\% [40/81] vs. $40 \%$ [17/43], $\mathrm{P}<0.05)$. Most patients required supplemental oxygen with noninvasive mechanical ventilation (52\%) and invasive mechanical ventilation (44\%) were also used in patients, among whom nonsurvivors accounted for a higher proportion and had worse illness. Extracorporeal membrane oxygenation was used in 5 patients; unfortunately, none of them survived. Regarding medications, glucocorticoids and antiviral drugs were widely used; interestingly, the use of glucocorticoids was statistically significant between the two groups $(P<0.05)$ and all kinds of antivirals showed no statistical difference. Not surprisingly, sepsis (100\% [57/57]) and acute respiratory distress syndrome (ARDS) $(91 \%$ [52/57]) were the two most common complications in nonsurvivors. Compared to survivors, nonsurvivors also showed a significantly higher proportion of secondary bacterial infection (74\% [42/57] vs. 25\% [17/67]), myocardial injury (51\% [29/57] vs. 17\% [11/67]), acute kidney injury $(65 \%[37 / 57]$ vs. $13 \%$ [9/67]), coagulopathy $(55 \%$ [35/57] vs. $6 \%[4 / 67])$ and abnormal liver function(51\% [29/57] vs. $12 \%[8 / 67])$.

As shown in Fig. 2, the dynamic changes in several key laboratory indexes were compared. It was apparent that TLC and PLT were generally lower in nonsurvivors than in survivors, whereas WBC and CRP levels were higher. WBC level fluctuated among nonsurvivors during the clinical course while it was under baseline condition among survivor. TLC levels were both at low standard throughout but the level of TLC gradually increased. As for CRP, the inflammatory indicator, overall level among nonsurvivors was remarkably higher than that in survivors. Moreover, biochemical indices of liver function, CK-MB, CRE and D-dimer did not differ significantly between two groups and the variations were small as a whole. (see Figure $\mathrm{E} 1$ in the online data supplement).

The distinct trajectories of temperature during the follow-up, which could provide valuable insight to monitoring the recovery of patients, are demonstrated in Fig. 3 . The patients were classified into 3 groups based on the temperature trajectory after admission as follows: group 1 (stabilized group; $n=69,55.65 \%$ ), 
maintained a stable temperature below $37^{\circ} \mathrm{C}$; group 2 (down-and-up group; $n=36,29.03 \%$ ), had a mean temperature that fell a little at the beginning and finally climbed up; and group 3 (steady-declining group; $n=19,15.32 \%$ ), the temperature of these patients was initially higher than normal and dropped gradually to approximately $36.6^{\circ} \mathrm{C}$ from the above normal body temperature. Patients with these 3 temperature trajectories had distinct fatalities. Group 1 had the lowest fatality rate at $21.74 \%$, with a mean temperature of $36.60{ }^{\circ} \mathrm{C}$. Patients in group $2 \mathrm{had}$ a mean temperature of $36.81{ }^{\circ} \mathrm{C}$ and a fatality rate of $69.44 \%$. In particular, the steady-declining group was characterized by the highest fatality rate of $84.21 \%$ (mean temperature, $37.44^{\circ} \mathrm{C}$ ). The characteristics at baseline and laboratory tests on admission stratified by temperature trajectory groups were presented in Table E1. Among group 3, the WBC, TLC, PLT and CRP levels were found to be significantly severer than the rest two. (see Table E1 in the online data supplement)

\section{Discussion}

Severe acute respiratory syndrome coronavirus 2 (SARS-CoV-2), which is genetically similar to the severe acute respiratory syndrome (SARS) coronavirus that resulted in the SARS outbreak in 2002, is responsible for a global pandemic of COVID-19. ${ }^{14}$ The outbreak began in China but has since spread around the world. ${ }^{15-17}$ The hospitals and clinics were initially overwhelmed, and both patients and healthcare providers became severely ill and died soon after. However, as the healthcare system response ramped up from the very beginning, more designated and newly built hospitals, like Wuhan Huoshenshan Hospital, and better equipment became available, the situation was under control within a short period in China. ${ }^{18}$

In our study, there were 124 confirmed cases of COVID-19 in the ICU of Wuhan Huoshenshan Hospital, and the 47 deaths resulted in a fatality rate of $46 \%$, which is close to the data reported by the Chinese Centers for Disease Control and Prevention. ${ }^{19}$ As mentioned in the previous literature ${ }^{19}$, approximately $80 \%$ of patients with COVID-19 have mild symptoms, and few people do not develop any symptoms or signs at all. For the remaining $20 \%$, as our study shows, these patients can range from mild symptoms, such as fever and dry cough, all the way to severe conditions, such as ARDS and even sepsis. Based on our data, the rate of critically ill patients is overwhelmingly male, overwhelmingly older. Similarly, both the rates of critical illness and the fatality rate are higher for patients with underlying health problems than for those without any of these conditions. In addition, many elderly patients typically have one or more of these conditions, so it is not surprising that these factors go hand in hand. Unlike seasonal flu, vaccines for SARS-CoV-2 have not yet been well established, although both are respiratory viruses. ${ }^{20}$ Therefore, elderly patients with underlying health problems are truly the ones at the highest risk.

The inflammation caused by SARS-CoV-2 builds up much fluid within and around the lung and results in ARDS and even subsequent multiple organ dysfunction syndrome, which means that the whole body starts shutting down. ${ }^{21}$ ARDS is a common complication during the clinical course and is the main cause of death. Among the nonsurvivors, all patients were diagnosed with ARDS and sepsis, which is often caused by severe infection. Our experience revealed that ARDS always had a sudden onset and rapid 
development, so few patients died soon. So far, not many treatment options exist for coronavirus, so the only option is offering supportive treatment, such as maintaining respiratory function with oxygen and ventilatory support, while the patient's immune system fights off the virus. Besides, multiple antiviral drugs have been tested, including arbidol hydrochloride, Lianhua Qingwen capsules and broad-spectrum antiviral agents. Unfortunately, none of them provide significant benefits. However, there are still debates about when and how to use glucocorticoids and whether patients should be incubated, though the results were statistically significant. In addition, it can be seen from Table 2 that the greatest need is a risk prediction model based on basic data at admission if the sample size is sufficient since many laboratory indexes are significantly different between survivors and nonsurvivors.

The dynamic changes in these biomarkers raise intriguing questions regarding the nature and extent of targeting of SARS-CoV-2. For example, obviously increased ALT/AST and TBIL occurred among patients with acute liver injury (37[30\%]), while those hepatic function indexes did not seem to change significantly overall and we supposed that the influence of underlying health problems cannot be ruled out. ${ }^{22}$ Nonetheless, we have reasons to believe that COVID-19-associated liver injury may be affected by various elements, such as hepatotoxic agents and a body-wide inflammatory reaction, and it may not be a common complication. ${ }^{13}$ Moreover, other statistically significant biomarkers, like WBC, TLC, PLT and CRP, are a compelling reminder for a poor prognosis. Numerically, TLC levels, which was expected based on the biological characteristics of the virus, dropped significantly among two groups in particular and nonsurvivors remained at a lower level.

Temperature trajectories reflect the potential dynamic changing patterns of temperature during the clinical course. We suppose that temperature is a consequence of infection and response of the body to it and patients in group 3 may be not able to fight against the virus in the later time of treatment. This observation provides new insights into COVID-19-associated temperature changes and indicates that critical patients with a high temperature should be particularly paid attention to by health care providers, especially those with high temperature on admission.

\section{Limitations}

On the other hand, our study has several limitations. First, the results of the arterial blood gas analysis, which we believe are quite valuable for prognosis, were incomplete due to strict controls involving blood samples; hence, we did not perform a thorough analysis. Another potential limitation is that we did not conduct further exploration based on findings (e.g. the difference between subgroups based on transfers) in this article because of the limited space of this thesis. Further studies that take these flaws and unanswered questions into account will need to be undertaken. ${ }^{23}$

\section{Conclusions}

The strengths of this study include that it is a large retrospective cohort study of critical patients in the ICU with information on COVID-19 and its associated outcomes from the first anti-pandemic frontline. It is 
worth mentioning that early in the disease, high temperature should receive extra care.

\section{Abbreviations}

COVID-19: Corona Virus Disease 2019

ICU: Intensive care unit

WHO: World Health Organization

PCT: Procalcitonin

WBC: White Blood Cell

TLC: Total lymphocyte count

PLT: Platelet

CRP: C-reactive protein

ALT: Alanine transaminase

AST: Aspartate transaminase

TBIL: Total bilirubin

hsTNI: hypersensitive troponin I

CRE: Creatinine

ARDS: Acute respiratory distress syndrome

SOFA: Sequential Organ Failure Assessment

IQR: Interquartile ranges

SARS-CoV-2: Severe acute respiratory syndrome coronavirus 2

SARS: Severe acute respiratory syndrome

\section{Declarations}

\section{Ethics approval and consent to participate}

This study was approved by the Ethics Commission of Wuhan Huoshenshan Hospital. (No. IEC-AF-SC-05) 
Informed consent was not obtained from the patients for this study due to the ongoing viral epidemic in mainland China at that time.

\section{Availability of data and materials}

Not applicable

\section{Competing interests}

There is no competing interest need to be declared.

\section{Funding/Support}

This study was supported by National Natural Science Foundation of China (Grant No. 81670015, $81722034,81988101,81830054,81802878$ ), the Ministry of Science and Technology Key Program (Grant No. 2018ZX09101002, 2017ZX100203205), Shanghai Pujiang Program (Grant No. 2019PJD059)

\section{Author's contributions}

WQL, XJZ and HT contributed to the study concept and design. YBR, JXW, WW and HT collected the original data. HSH, YSM, XWQ and TYS compiled the data. HSH, ZCJ, WW, HYW, WQL, XJZ and HT analyzed and interpreted the data. HSH wrote the draft manuscript. ZCJ and CXZ offered the statistical analyses. SZC, WW, JXW and HT revised the manuscript for important intellectual content. SZC, WW, HT and HYW obtained the funding. YBR, WW, XJZ, WQL, HT and HYW provided administrative support. XJZ, WQL, HT and HYW supervised the project and gave final approval.

\section{Acknowledgements}

We thank all of the patients, their families and all medical staff involved in this battle against SARS-CoV2.

\section{References}

1. Huang C, Wang Y, Li X, et al. Clinical features of patients infected with 2019 novel coronavirus in Wuhan, China. Lancet. 2020;395(10223):497-506. doi:10.1016/S0140-6736(20)30183-5

2. Guan W-J, Ni Z-Y, Hu Y, et al. Clinical Characteristics of Coronavirus Disease 2019 in China. N Engl J Med. Published online February 28, 2020. doi:10.1056/NEJMoa2002032

3. National Health Commission of the People's Republic of China. Accessed October 10, 2020. http://www.nhc.gov.cn/

4. Clinical management of severe acute respiratory infection when novel coronavirus (nCoV) infection is suspected. Accessed March 23, 2020. https://www.who.int/publications-detail/clinicalmanagement-of-severe-acute-respiratory-infection-when-novel-coronavirus-(ncov)-infection-issuspected 
5. New coronavirus pneumonia prevention and control program (7th ed.). Accessed March 23, 2020. http://www.nhc.gov.cn/yzygj/s7653p/202003/46c9294a7dfe4cef80dc7f5912eb1989.shtml

6. Chen N, Zhou M, Dong X, et al. Epidemiological and clinical characteristics of 99 cases of 2019 novel coronavirus pneumonia in Wuhan, China: a descriptive study. :7.

7. Wang D, Hu B, Hu C, et al. Clinical Characteristics of 138 Hospitalized Patients With 2019 Novel Coronavirus-Infected Pneumonia in Wuhan, China. Published online 2020:9.

8. Wu C, Chen X, Cai Y, et al. Risk Factors Associated With Acute Respiratory Distress Syndrome and Death in Patients With Coronavirus Disease 2019 Pneumonia in Wuhan, China. Published online 2020:10.

9. Yang X. Clinical course and outcomes of critically ill patients with SARS-CoV-2 pneumonia in Wuhan, China: a single-centered, retrospective, observational study. :7.

10. Zhou F, Yu T, Du R, et al. Clinical course and risk factors for mortality of adult inpatients with COVID19 in Wuhan, China: a retrospective cohort study. :9.

11. ARDS Definition Task Force, Ranieri VM, Rubenfeld GD, et al. Acute respiratory distress syndrome: the Berlin Definition. JAMA. 2012;307(23):2526-2533. doi:10.1001/jama.2012.5669

12. Singer $M$, Deutschman CS, Seymour CW, et al. The Third International Consensus Definitions for Sepsis and Septic Shock (Sepsis-3). JAMA. 2016;315(8):801-810. doi:10.1001/jama.2016.0287

13. Chinese Digestion Association CMDA, Chinese Society of Hepatology CMA. The protocol for prevention, diagnosis and treatment of corona virus infective disease 2019. Chinese Journal of Hepatology. 2020;28(00):E004-E004. doi:10.3760/cma.j.cn501113-20200309-00095

14. Lu R, Zhao X, Li J, et al. Genomic characterisation and epidemiology of 2019 novel coronavirus: implications for virus origins and receptor binding. Lancet. 2020;395(10224):565-574. doi:10.1016/S0140-6736(20)30251-8

15. Livingston E, Bucher K. Coronavirus Disease 2019 (COVID-19) in Italy. JAMA. Published online March 17, 2020. doi:10.1001/jama.2020.4344

16. Day M. Covid-19: surge in cases in Italy and South Korea makes pandemic look more likely. BMJ. 2020;368:m751. doi:10.1136/bmj.m751

17. Holshue ML, DeBolt C, Lindquist S, et al. First Case of 2019 Novel Coronavirus in the United States. $N$ Engl J Med. 2020;382(10):929-936. doi:10.1056/NEJMoa2001191

18. Wu Z, McGoogan JM. Characteristics of and Important Lessons From the Coronavirus Disease 2019 (COVID-19) Outbreak in China: Summary of a Report of 72314 Cases From the Chinese Center for Disease Control and Prevention. JAMA. Published online February 24, 2020. doi:10.1001/jama.2020.2648

19. Response EWG for NE, Prevention CC for DC and. The epidemiological characteristics of an outbreak of 2019 novel coronavirus diseases (COVID-19) in China. Chinese Journal of Epidemiology. 2020;41(02):145-151. doi:10.3760/cma.j.issn.0254-6450.2020.02.003 
20. Walls AC, Park Y-J, Tortorici MA, Wall A, McGuire AT, Veesler D. Structure, Function, and Antigenicity of the SARS-CoV-2 Spike Glycoprotein. Cell. Published online March 6, 2020.

doi:10.1016/j.cell.2020.02.058

21. Xu Z, Shi L, Wang Y, et al. Pathological findings of COVID-19 associated with acute respiratory distress syndrome. Lancet Respir Med. Published online February 18, 2020. doi:10.1016/S22132600(20)30076-X

22. Chai X, Hu L, Zhang Y, et al. Specific ACE2 Expression in Cholangiocytes May Cause Liver Damage After 2019-nCoV Infection. :13.

23. Callaway E, Cyranoski D. China coronavirus: Six questions scientists are asking. Nature. 2020;577(7792):605-607. doi:10.1038/d41586-020-00166-6

\section{Figures}

Critical patients admitted to ICU
from $8^{\text {th }}$ February to 16 th March
$(n=126)$

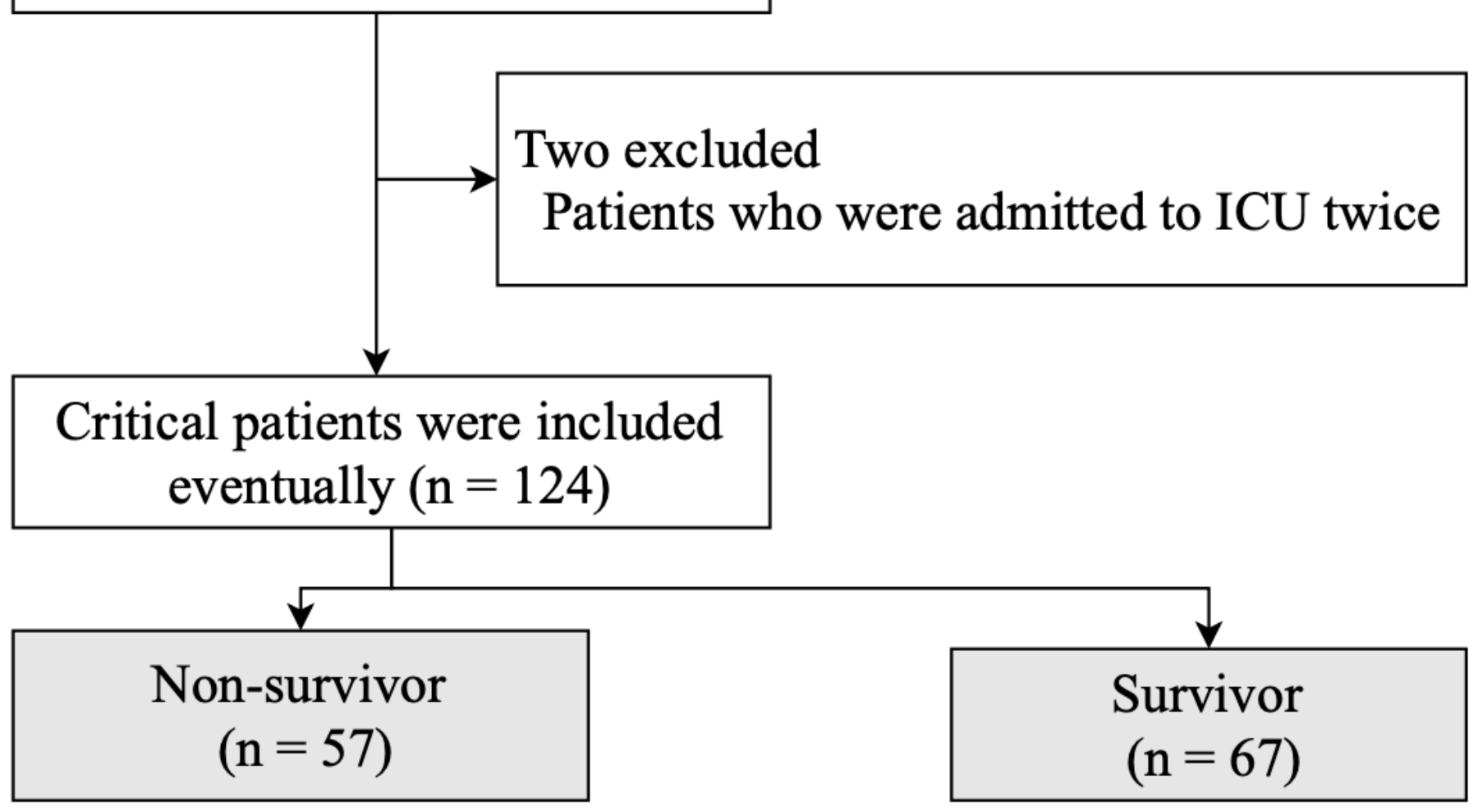

Figure 1

Flow chart of patient inclusion 
A
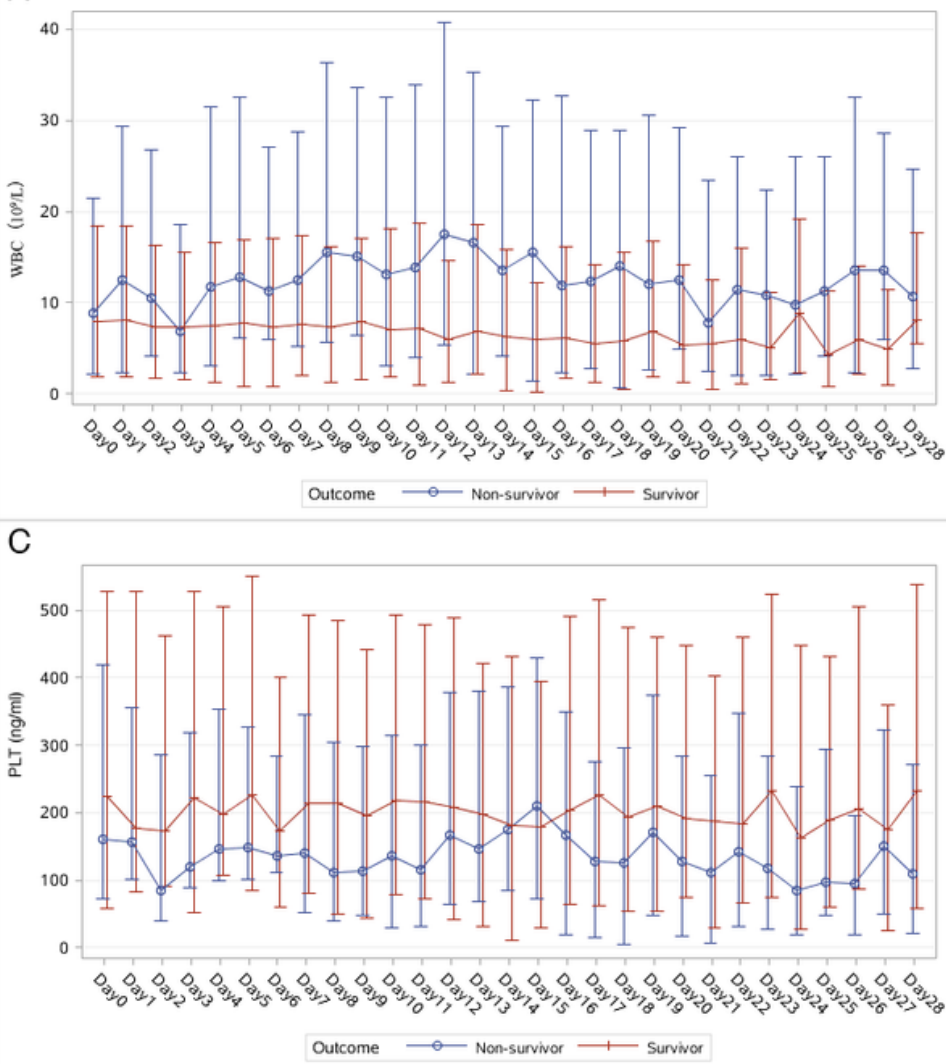

B

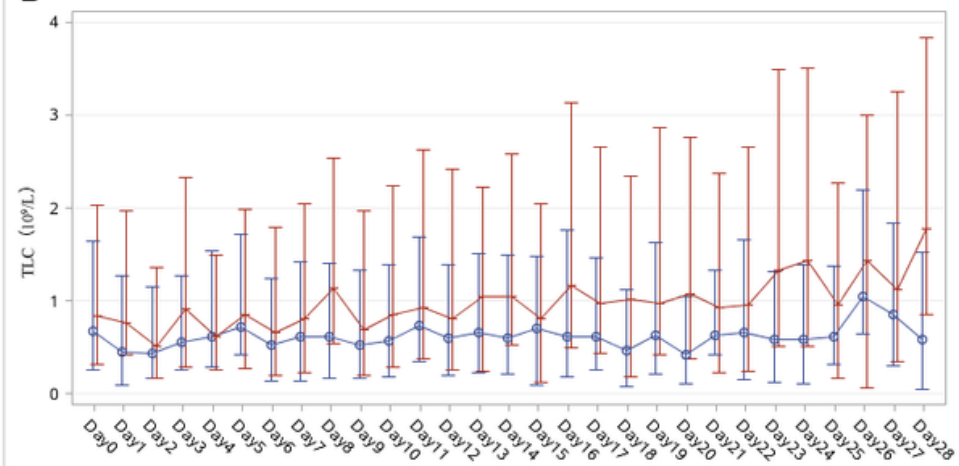

Outcome -0 Non-survivor 1 Survivor

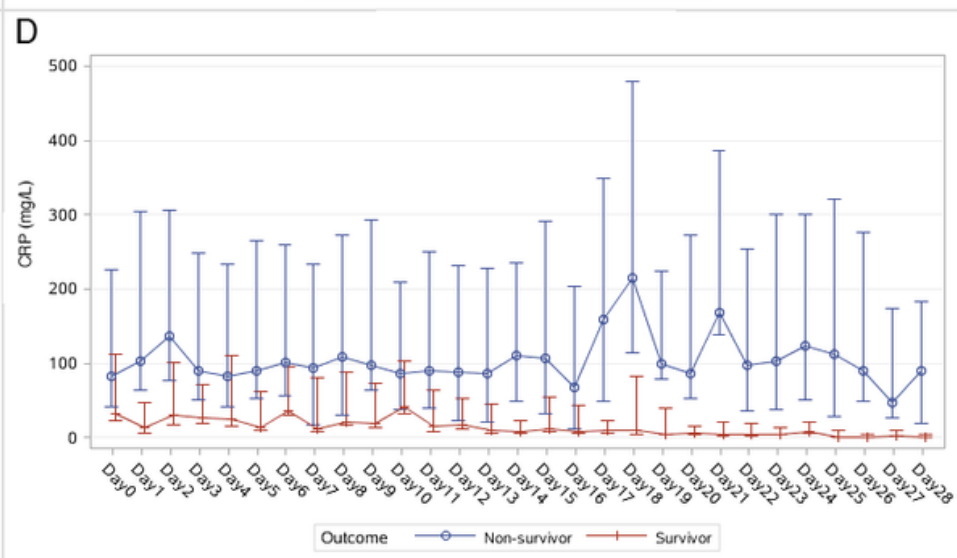

Figure 2

Dynamic changes of several important laboratory indexes during the follow-up period. (A) White blood cell (WBC) counts; (B) Total Lymphocyte Count (TLC); (C) Platelet (PLT); (D) C-reactive protein (CRP); 


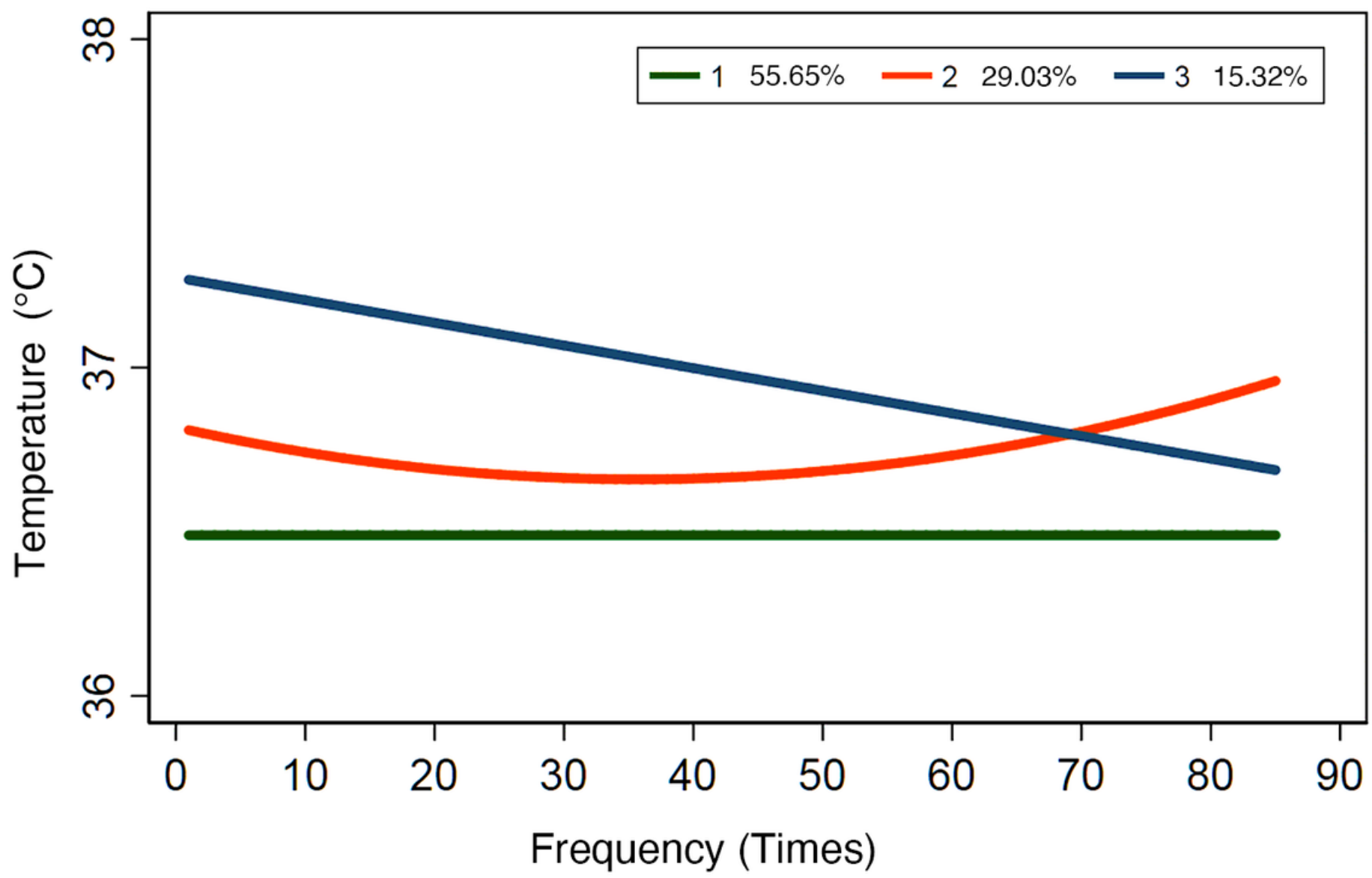

Figure 3

Trajectories of temperature for patients from admission to 16th April. Group 1 (a stabilized group; $n=69$, 55.65\%); Group 2 (a down-and-up group; n=36, 29.03\%); Group 3 (a steady-declining group; n=19, 15.32\%)

\section{Supplementary Files}

This is a list of supplementary files associated with this preprint. Click to download.

- FigureE1.tif

- TableE1.docx 